\title{
Arqueología Histórica en contextos de trasformación urbana. El caso de Concepción de la Sierra (Misiones, Argentina). Entre la materialidad arqueológica y la patrimonialización de un pueblo misionero
}

\section{Amanda E. Ocampo*}

Fecha de defensa: 19 de mayo de 2020

Director: Dr. Daniel Loponte

Jurados: Dres. Mariano Ramos, Carolina Rivet y Victoria Roca

\section{Introducción}

Este trabajo de tesis doctoral propuso el estudio del sitio arqueológico de la antigua reducción de "Nuestra señora de la Concepción de la limpia Ibitiracuá" o Concepción, ubicado en el casco urbano del municipio de Concepción de la Sierra en la provincia de Misiones. El mismo se posiciona como un espacio de gran potencial para investigar y aportar al estudio de la historia del periodo jesuítico desde la arqueología, contribuyendo además a la protección del patrimonio histórico y arqueológico de la región desde una perspectiva interdisciplinaria.

Como punto de partida se introdujo una revisión general de los pueblos de la actual provincia de Misiones; tanto de trazados urbanos jesuíticos como de los posteriores, pensando en las circunstancias de ocupación y asentamiento que influyeron en los procesos de reorganización espacial como consecuencia de la expulsión de los jesuitas (Poenitz y Poenitz, 2017). En este sentido, se comenzó por el momento de ocupación jesuítica como marco contextual y con el objetivo de identificar características particulares en la dinámica de reutilización de los sitios en perspectiva histórica, puesto que algunos ex- pueblos jesuíticos están resituados y reactualizados en contextos modernos.

\section{Planteo del problema, objetivos de investigación e hipótesis}

Teniendo en cuenta una primera aproximación al contexto general del casco histórico del pueblo es que se reconoció una materialidad de un sitio arqueológico reduccional en un espacio habitado. A partir de este punto de partida es que nos preguntamos qué lugar jugaron dichos materiales en la dinámica diaria del pueblo, como se manifestaron a lo largo del tiempo, y si constituyeron (o no) parte de las transformaciones propias de un territorio en constante movimiento. Se propuso observar y registrar el estado actual de un pueblo en tanto patrimonio

* Secretaria de Investigación y Postgrado (SlyP), Facultad de Humanidades y Ciencias Sociales, Universidad Nacional de Misiones (UNaM) - Consejo Nacional de Investigaciones Científicas y Técnicas (CONICET). Tucumán 1946 (CP N3300BSP) Posadas, Misiones, Argentina. E-mail: amiocampo25@gmail.com arqueológico de la historia entre jesuitas y guaraníes, vinculado a los rasgos y las transformaciones de dicho lugar. Desde los estudios arqueológicos se planteó analizar la existencia y persistencia de los materiales propios del período reduccional situado en un sitio en pleno contexto urbano, insertos en territorios socio-espaciales dinámicos, activos y cambiantes, y constitutivos de un pueblo misionero de la actualidad.

\section{Objetivos generales}

»Definir, a partir de estudios arqueológicos, una metodología de investigación que articulara procesos de patrimonialización con una comprensión de los procesos sociales y de resignificación de la antigua reducción jesuítica Concepción.

»Establecer pautas teórico-metodológicas propias de una Arqueología Histórica en contextos de transformación urbana en sitios reduccionales de la región.

\section{Objetivos específicos}

» Realizar un registro de materiales arqueológicos a partir de trabajos de prospección, excavaciones arqueológicas y laboratorio.

"Analizar la reutilización de los espacios que se encuentran dentro del casco histórico del pueblo de Concepción de la Sierra, articulando vías de información arqueológica e histórica en relación con elementos propios de un sitio habitado en la actualidad.

» Reconocer la conexión pasado-presente en los espacios reutilizados que se presentaron a través de la examinación de los elementos estructurales y materiales evidenciados en el contexto arqueológico y moderno actual.

Además, el trabajo de investigación y sus resultados tuvieron como objeto: a) generar un aporte que definiera un enfoque práctico para la observación y estudio de otros pueblos que conformaron el conjunto jesuítico-colonial y b) integrar la lectura arqueológica, tanto como una herramienta de activación patrimonial y de rescate de las manifestaciones materiales así situadas, como del estado de los materiales arqueológicos en los sitios reduccionales. Este enfoque que conjuga la arqueología de contextos históricos y la patrimonialización de reducciones jesuíticas, es fundamental para resaltar el 
potencial del pueblo como capital cultural comunitario y promover la salvaguarda de la memoria de las misiones jesuíticas en general. A partir de los objetivos propuestos se plantearon las siguientes hipótesis de investigación:

» El casco histórico del actual pueblo presentó un desarrollo urbano que, a pesar de no cumplir las mismas funciones sociopolíticas que se encontraban en la antigua reducción, conservaron elementos funcionales y formales similares al trazado del periodo colonial inserto en el contexto actual.

» La interacción entre guaraníes y un nuevo modelo de vida impuesta por la Compañía de Jesús se reflejó en el registro arqueológico combinando estilo y decoración, cuyas funciones y estructura distribucional reflejaron actividades desarrolladas en el área del sitio.

» La población local habitó un patrimonio arqueológico que activaba una memoria social vinculada a un determinado periodo histórico de los pueblos de jesuitas y guaraníes y manifestada a partir de una materialidad arqueológica circundante y presente en la cotidianeidad de una comunidad actual.

\section{Características generales del área de estudio}

Se indica además que la tala y rozado redujeron la superficie del bosque que hoy subsiste en sitios de difícil acceso, en parcelas de propiedad fiscal y a lo largo de arroyos; en medio

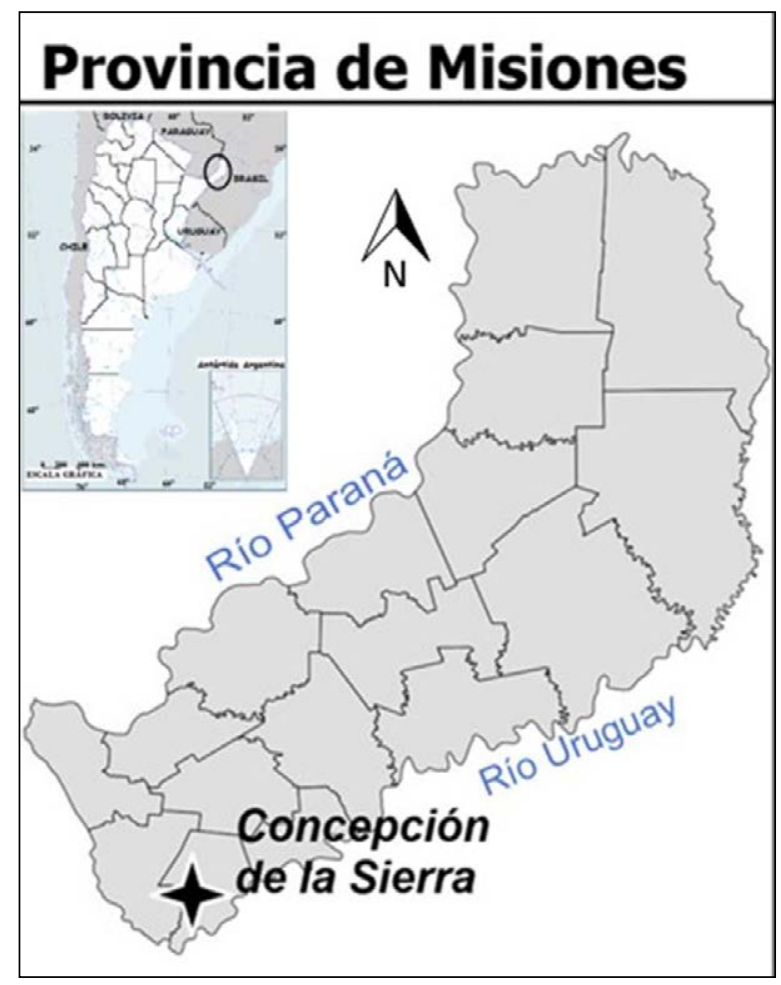

Figura 1. Ubicación del área de estudio. de los pajonales mesófilos se encuentran como testimonio isletas de bosque de distinto tamaño (Rodríguez, Cardozo, Ruiz Díaz, Darien y Prado, 2006). Se debe sumar además a las características ambientales el avance continuo de las actividades antrópicas sobre el medio natural, tales como obras propias del avance urbano y poblacional, zonas dedicadas al cultivo de la yerba mate y otras explotaciones forestales y agropecuarias de la región (Lewczuk, 2009).

\section{Marco teórico de investigación: arqueología histórica, patrimonio y región}

Se planteó un abordaje teórico que habilitara tratar diversos aspectos, dimensiones y territorialidades de los espacios históricos de las reducciones. Como primer paso se propuso a la Arqueología Histórica en contextos de transformación urbana, para posteriormente articular dicho enfoque con una propuesta de patrimonio arqueológico y estudios regionales.

La arqueología histórica puede definirse como "el ámbito de las ciencias que aborda el problema del pasado humano ubicado en tiempos históricos y que puede utilizar para su resolución, información proveniente del registro arqueológico y de documentos escritos" (Ramos, 2002, p. 646).

El registro arqueológico sobre el sitio y su contexto está orientado a interpretar las conexiones históricas desde la materialidad jesuítica-guaraní para comprender tales manifestaciones materiales como productos del pasado que interpelan las acciones actuales situadas en un contexto histórico en constante transformación. Se destaca así la existencia de un patrimonio arqueológico, integrado al devenir cotidiano de su gente (Senatore, 2004); en el que confluye una arquitectura redefinida en espacios diferenciados en los que se evidencian rasgos elementales de las experiencias de la vida en reducción.

Los sitios arqueológicos van cambiando, transformando y resignificándose a lo largo del tiempo, constituyéndose en un paisaje testigo y/o protagonista de procesos sociales $y$ trayectorias que van forjando identidades empoderadas por los grupos humanos que los manipulan.

Se propone pensar al patrimonio en referencia a lo que existe y se construye, en donde cada actor social se relaciona con sus patrimonios mediante distintas prácticas empíricas y cognoscitivas, objetos, espacios y tiempos a partir de experiencias y acciones en las múltiples esferas del plano social (Alonso González, 2016). Atender patrimonios arqueológicos en términos de relaciones sociales que existen en y a través de nuestros mundos materiales (Miller, 2005).

A partir de centrar la investigación en una de las reducciones que fueron parte del sistema misionero de los 30 pueblos de jesuitas y guaraníes, es que sumamos al enfoque teórico 
una perspectiva regional, que pone el punto de interés en los estudios regionales como objeto de análisis.

Teniendo en cuenta que el espacio de CS dispone de una materialidad arqueológica que une un pasado con un presente, es que los estudios regionales ofrecen una alternativa para estudiar en una misma sociedad "aquello que perdura y aquello que cambia” (Auge, 1998, p.14) a partir de lo que fueron los espacios reduccionales y su continuidad histórica. Al analizar el caso puntual de dicho pueblo, se encuentran y reconocen una diversidad de experiencias concentradas en rastros que se superponen, en parte hermetizados y en parte permeables a la modificación.

\section{Metodología de trabajo}

Se recurrió a un conjunto de métodos y técnicas tanto arqueológicas como etnográficas, que nos permitieron abordar la temática de estudio. Tales tareas se organizaron en trabajos de gabinete, campo y laboratorio.

» Gabinete: búsqueda de antecedentes bibliográficos.

"Campo: entrevistas con habitantes de la zona, prospecciones delimitando un área específica y dividiendo las tareas prospectivas en $\mathrm{N}-\mathrm{S}$ y E-O, y trabajos de excavación, realizados en seis loci o puntos específicos en el espacio, identificados a partir del plano de la reducción y la disponibilidad de los lugares.

" Laboratorio: tareas de identificación, cuantificación y clasificación de los materiales arqueológicos recuperados, y un registro de sus características macroscópicas para la creación de una base de datos. Los materiales analizados fueron divididos en las categorías de: cerámica (subdividida en tejas, cerámica indígena, hispano-indígena y europea), vidrio, líticos y metal.

\section{Resultados generales}

A partir del análisis de distintas líneas de evidencia generadas a lo largo de la investigación se discuten datos que permiten interpretar al área de estudio como un sistema complejo y dinámico que se fue transformando a lo largo del tiempo. Para ello se presentan a modo de ejes conductores de discusión, tres temas fundamentales: sitio arqueológico, reutilización y patrimonialización.

En primer lugar, el casco histórico de CS presenta un sitio arqueológico reduccional con un pueblo circundante y actividades diarias que generan obras y nuevas construcciones, movimiento del suelo y distintas transformaciones en el terreno generando una pérdida de contextos arqueológicos. Se suman además las características ambientales locales, en donde los altos contenidos de humedad, lluvias periódicas y suelos arcillosos generan un bajo estado de conservación o directa pérdida de materiales.

No obstante, y a pesar de estos elementos condicionantes, durante las tareas de prospección realizadas se registró una continuidad y transformación del paisaje urbano que involucra la materialidad arqueológica del periodo reduccional. El rasgo arqueológico principal son los muros y cimientos construidos con rocas itacurú como recurso local, indicando un aprovechamiento intensivo de la materia prima, levantada y usada en la actualidad y el cual habría sido parte del trazado urbanístico de la antigua reducción.

Si bien hubo determinadas limitaciones en cuanto a definir una precisión cronológica para ordenar distintos momentos históricos de ocupación, teniendo en cuenta además la reutilización espacial del área, se identificaron locus con variedad de material arqueológico que sobrevivieron al paso del tiempo e indican un sitio con un alto índice de restos insertos en contextos urbanos.

Los hallazgos realizados durante los trabajos de excavación también muestran evidencia de los periodos de saqueo, destrucción y pérdida de la reducción. También se registraron elementos que dan cuenta de periodos posteriores a la expulsión y nuevas ocupaciones.

En relación con el periodo reduccional contamos en primera instancia con el material constructivo de tejas. Es el material más abundante encontrado en todas las áreas excavadas y a lo largo de todo el pueblo. Las mismas fueron utilizadas para la realización de techos de viviendas y edificios públicos de la reducción.

Los restos de cerámica fueron uno de los hallazgos más frecuentes y diagnósticos, indicando evidencias de actividad hispano indígena y procesos de aculturación a partir del encuentro entre distintos estilos y técnicas de trabajar la materia prima local.

También se registraron otros elementos constructivos que otorgaron indicios cronológicos relativos tales como columnas, capiteles y bases que actualmente se encuentran en la Casa de la Cultura de CS. Otros restos analizados fueron de lozas, mayólica, gres y porcelana, posiblemente introducidos en un momento posterior a la expulsión de los jesuitas, en un periodo de consolidación y expansión industrial capitalista europea del siglo XIX (Fernetti, s/f), atendiendo a una creciente circulación de objetos que llegaban desde los grandes centros de producción europeos. En cuanto al registro de restos vítreos, si bien también contamos solo con fragmentos menores, podemos inferir que habrían sido parte de botellas de bebidas alcohólicas y de contextos modernos del siglo XIX y XX. 
En cuanto al proceso de reutilización, el mismo se define como un proceso de reaprovechamiento de un espacio o un elemento material. En nuestro caso de estudio nos encontramos con un espacio de planificación urbana típica de una antigua reducción jesuítica, la cual en un periodo posterior, pasa por un proceso de reciclaje y es reutilizado a los fines del nuevo pueblo, desarrollando un nuevo modelo urbano, con funciones similares a su antigua ocupación. Los espacios reutilizados muestran una articulación entre sitios arqueológicos y la dinámica de los pueblos actuales.

Se enfatiza así la idea de que los procesos de reutilización fueron del tipo material y espacial, generando ambos un proceso de patrimonialización en el cual se introduce una comunidad local, activa y poseedora capaz de continuar y adecuarse a una refuncionalización que tuvo sus principios en un pasado histórico. Lejos de un único contexto, la materialidad arqueológica atraviesa diversos lugares y sentidos (Petrosini, 2018).

De esta manera el proceso de patrimonialización se manifiesta desde un proceso histórico mediado por su reutilización. Dicha acción llevó al desarrollo de prácticas compartidas relacionadas con el uso del paisaje y que se hacen presentes en las formas actuales en que pasado y paisaje son vividos y representados (Salerno, 2016) (Figura 2).

\section{Sobre Historias regionales: sitio reduccional, memoria material}

La propuesta de pensar en un sitio reduccional y su proceso de patrimonialización inserto en un pueblo de Misiones propuso indagar en la complejidad del sitio arqueológico en cuanto a su conexión pasado colonial- presente patrimonial.
Hay una memoria manifiesta que en este caso no es solo una fuente de conocimiento sino también un modo de experimentar el entorno (Ramos, 2011). Su carácter de palimpsesto, memoria viva de un pasado ya muerto, transforma el paisaje en instrumento de trabajo que permite ver las etapas del pasado con una perspectiva de conjunto (Bloch, 1974; Santos, 1996).

En definitiva, los relatos orales y la materialidad histórica registrada presente en el pueblo son formas conmemorativas de la memoria. Su espacio circundante actúa como una "semilla de rememoración", como recuerdo, y para recordar siempre debe haber algo de ese pasado. El conocimiento histórico de la comunidad, acompañado de una materialidad arqueológica evidenciada sirven como recursos mnemotécnicos (Rappaport, 2005) para recordar la historia del periodo reduccional jesuíticoguaraní.

Este gran corpus de información nos alienta a seguir trabajando en una línea de investigación teniendo en cuenta la singularidad del sitio, su registro y estado actual. Armar una propuesta que implique realizar acciones vinculadas al rescate de la memoria del pueblo y su patrimonio.

Finalmente, no queda nada más que decir que en esta investigación se propuso reflexionar acerca de cómo los sitios arqueológicos y su patrimonialización no deben generar acciones dirigidas a inmovilizar, congelar, o fosilizar determinados momentos culturales (Smith, 2011) sino que los mismos nos invitan a que como arqueólogas/os podamos ver la continuidad histórica y sus constantes movimientos, cambios y resistencia a ser olvidados.

\section{Referencias citadas}

"Alonso González, P. (2016). Patrimonio y ontologías

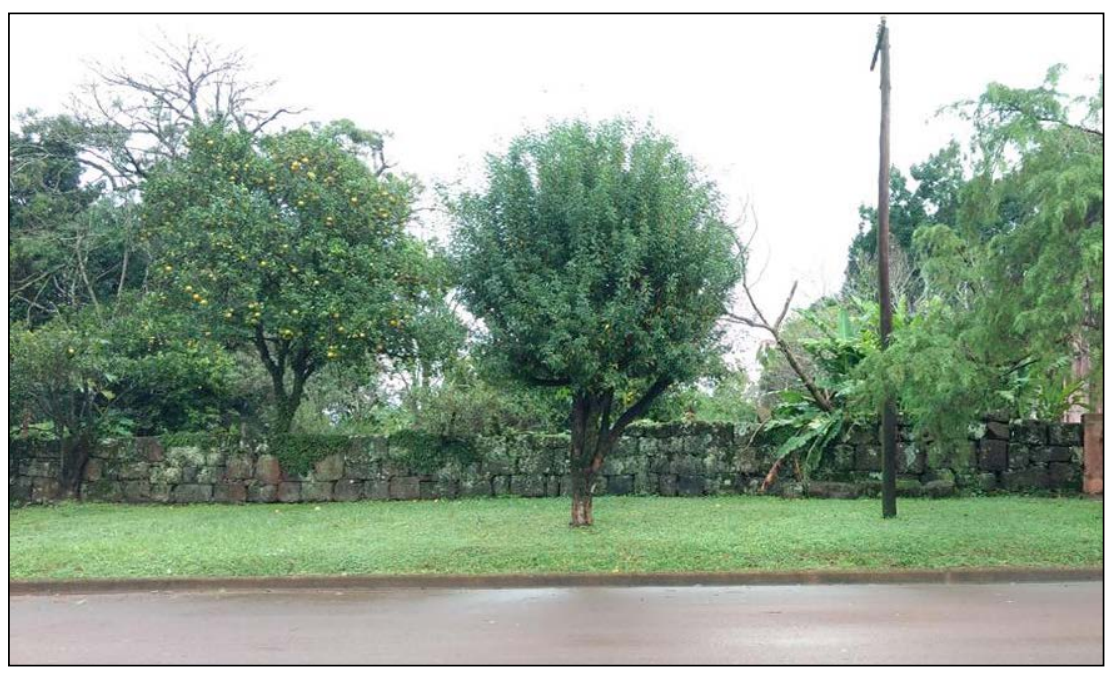

Figura 2. Muro de rocas itacurú que forma parte del frente de una vivienda particular. 
múltiples: hacia la co-producción del patrimonio cultural. En C. Gianotti García, D. Barreiro Martínez y B. Vienni Baptista (Eds.), Patrimonio y Multivocalidad. Teoría, Práctica y Experiencias en torno a la Construcción del Conocimiento en Patrimonio (pp.179-198). Montevideo: Universidad de la República y Consejo Superior de Investigaciones Científicas (CSIC).

》Auge, M. (1998). El espacio histórico de la Antropología y el tiempo antropológico de la historia. En M. Auge (Ed.), Hacia una Antropología de los Mundos Contemporáneos (pp. 1130). Barcelona: Gedisa.

"Bloch, M. (1974). Apología de la Historia. Barcelona: Empuries.

》 Fernetti, G. (s/f). Descripción de la técnica transferware (1780-1920). Un resumen usando cerámicas arqueológicas de Rosario, Argentina. Centro de Estudios de Arqueología Histórica (CEAH), Universidad Nacional de Rosario, Argentina. Manuscrito inédito.

" Lewczuk, S. R. (2009). La selva, producto turístico del destino emergente Yaboti (Tesis de pregrado inédita), Universidad Nacional de Misiones, Argentina.

" Miller, D. (2005). Materiality: An introduction. En D. Miller (Ed.), Materiality (pp. 1-50). Durham: University Press.

"Petrosini, A. (2018). Agencia y patrimonio jesuíticoguaraní. Una biografía de la circulación de restos materiales en museos de Córdoba y Misiones a partir de la segunda mitad del siglo XX. En E. C. Deckman Fleck y J. H. Rogge (Eds.), La Acción Global de la Compañía de Jesús Acao Global da Companhia de Jesus: Embajada Política y Mediación Cultural en un Escenario Mundial (pp. 9711018). Sao Leopoldo: Oikos.

»Ramos, A. (2011). Perspectivas antropológicas sobre la memoria en contextos de diversidad y desigualdad. Alteridades, 21(42), 131-148. https://alteridades.izt.uam. $\mathrm{mx} /$ index.php/Alte/article/view/119

» Ramos, M. (2002). El proceso de investigación en la denominada Arqueología Histórica. En D. Schavelzon, V. Duran y H. Schiavazza H. (Eds.), Arqueología Histórica Argentina. Actas del Primer congreso Nacional de Arqueología Histórica (pp. 645-658). Buenos Aires: Corregidor.

» Rappaport, J. (2005). Cumbe Renaciente. Una Historia Etnográfica Andina. Bogotá: Instituto Colombiano de Antropología e Historia.

» Rodríguez, M. E., Cardozo, A., Ruiz Díaz, M., Darien, E. y Prado, D. E. (2006). Los bosques nativos misioneros: estado actual de su conocimiento y perspectivas. En M. Arturi, J. Frangi y J. Goya (Eds.), Ecología y manejo de los bosques de Argentina (pp. 3-33). La Plata: Editorial de la Universidad de la Plata (EDULP).

»Salerno, V. (2016). Apropiación de objetos arqueológicos en la microrregión del río Salado Bonaerense. Revista de Antropología del Museo de Entre Ríos, 2(2), 93-97. https://ppct.caicyt.gov.ar/index.php/antropmuser/article/ view/10323

»Santos, M. (1996). La naturaleza del Espacio. Técnica y Tiempo. Razón y Emoción. Barcelona: Ariel.

"Senatore, X. (2004). Discursos ilustrados y sociedad moderna en las colonias españolas de Patagonia. En A. Zarankin y P. P Funari (Eds.), Arqueología Histórica en América del Sur; los Desafíos del S. XXI (pp. 29-54). Bogotá: Universidad de Los Andes.

»Smith, L. (2011). El "espejo patrimonial”. ¿llusión narcisista o reflexiones múltiples? Antípoda. Revista de Antropología y Arqueología, 12, 39-63. https://doi.org/10.7440/ antipoda12.2011.04 